\title{
FORMACIÓN PARA LA INVESTIGACIÓN: UNA APROXIMACIÓN A LA CULTURA INVESTIGATIVA Y CONOCIMIENTO CONTABLE
}

\author{
TRAINING FOR RESEARCH: AN \\ INVESTIGATIVE APPROACH TO CULTURE \\ AND STOCKHOLDERS KNOWLEDGE
}

\author{
Ofelia Gómez Niño', Carmen Luz Cely López²
}

\section{RESUMEN}

La educación superior en Colombia, presenta cambios importantes orientados al aseguramiento de la calidad y pertinencia. Con la Ley 30 de 1992 se dieron las bases sobre las que se construye el sistema de aseguramiento de la calidad de la educación superior; así mismo define el campo normativo, establece el principio de calidad en el servicio, promueve la creación de grupos asesores como el Consejo Nacional de Educación (CESU); reglamenta las funciones del Instituto Colombiano de Fomento a la Educación Superior (ICFES), contempla acciones de evaluación docente, considera la creación de organismos como el Sistema Nacional de Acreditación y sistemas de información.

Conscientes de la responsabilidad en la formación contable, es necesario alinearla a las exigencias del Estado, sector productivo y la sociedad. El objetivo de esta investigación se orientó a identificar la cultura investigativa en procesos de formación académica y generación de conocimiento en ciencias contables en los programas de Contaduría Pública de Instituciones de Educación Superior ubicadas en el Área Metropolitana de Bucaramanga. El alcance se centró en dar una mirada a los actores académicos y currículos de programas con trayectoria e impacto en la región, para saber cómo perciben estudiantes, profesores y decanos, la calidad de la educación, la generación de conocimiento y la investigación.

Metodológicamente, el estudio se sustenta en el paradigma cualitativo-cuantitativo, descriptivo con enfoque etnográfico; en la recolección de información se aplicó un cuestionario a estudiantes y profesores, entrevistas semi-estructuradas a decanos y se observó el Proyecto Educativo Institucional (PEI), lineamientos curriculares. Los resultados destacan que los estudiantes ven la investigación importante, pero se resisten a ella; la participación en investigación es escasa, presentan debilidades en hábitos de lectura y escritura; los profesores poseen fortalezas en la visión investigativa; ejercitan el pensamiento crítico, fomentan actividades de trabajo en equipo y aprendizaje significativo.

Palabras claves: Conocimiento contable, cultura, educación, formación, investigación.

Recepción: 17/01/2017. Aprobación: 11/10/2017.

\section{ABSTRACT}

Higher education in Colombia, presents significant changes aimed at ensuring the high quality level and relevance. With Law 30 of 1992, the basis on which the system of quality assurance in

1 Universidad Cooperativa de Colombia sede Bucaramanga, ofelia.gomez@campusucc.edu.co

2 Universidad Cooperativa de Colombia, Universidad Nacional de Colombia, Bogotá, carmen.cely@campusucc.edu.co 
higher education is built occurred; It also defines the legal field, establishes the principle of quality service, promotes the creation of advisory groups such as the National Council of Education (CESU); regulates the functions of the Colombian Institute for the Promotion of Higher Education (ICFES), teacher's evaluation, consider the creation of bodies such as the National Accreditation System and information systems.

Aware of the responsibility in accounting education, it is necessary to align the requirements of the State, the productive sector and society. The objective of this research was aimed at identifying the research culture in processes of academic training and knowledge generation in Accounting programs in Public Institutions of Higher Education located in the Metropolitan Area of Bucaramanga. The scope focused on giving a look to academic programs and curriculum trajectory and impact in the region, to know how perceive students, teachers and deans, quality of education, knowledge generation and research actors.

Methodologically, the study is based on qualitative and quantitative, descriptive paradigm with ethnographic approach; gathering information in a questionnaire to students and teachers, semistructured interviews to deans and the Institutional Education Project (PEI) was observed curricular guidelines interviews was applied. The results highlight that students see research as something important but resist it; participation in research is limited, have weaknesses in reading and writing habits; teachers have strengths in vision research; exercise critical thinking, encourage teamwork activities and meaningful learning.

Keywords: Accounting, education, knowledge, research, training.

\section{INTRODUCCIÓN}

La educación es un acto intencionado, es ante todo una acción; significa que para que algo sea considerado educativo debe estar mediado necesariamente por la conciencia y la voluntad de quien ejecuta el acto (Unigarro, 1999). El verdadero sentido de la educación se evidencia cuando es factible formar individuos conscientes para actuar en las áreas de formación o disciplinas afines. La formación va moldeando aspectos relacionados con la cultura, entendida como aquello que refiere conductas, actitudes y pensamientos del hombre aprendidos en un grupo social. Como lo expresa (Geertz, 1994), observar la cultura no como una entidad, sino como "algo a lo que puedan atribuirse de manera casual acontecimientos sociales y modos de conducta; la cultura es un contexto dentro del cual pueden describirse todos los fenómenos de manera inteligible, es decir "densa". Comprender la cultura de un pueblo supone captar su carácter normal sin reducir su particularidad".

En educación se requiere generar cultura en el desarrollo de habilidades investigativas, descubrir los saberes esenciales para elevar las fronteras del conocimiento. Como lo expone (Restrepo, 2003), la investigación está asociada con la calidad de la educación superior; la investigación formativa es una primera aproximación a la existencia de una cultura que se manifiesta de dos maneras: enseñar a investigar y hacer investigación. La pregunta que orientó la investigación es ¿Cuál es la cultura investigativa en procesos de formación académica y generación de conocimiento en ciencias contables de los programas de Contaduría Pública de Instituciones de Educación Superior ubicadas en el Área Metropolitana de Bucaramanga? Los propósitos expresados por (Geertz, 1994) son fundamentales, al observar la cultura no como una entidad, sino como "algo a lo que puedan atribuirse de manera casual acontecimientos sociales y modos de conducta; entonces, la cultura implica entenderla como un proceso de comprensión entre comunidades.

En la academia expone (Restrepo, 2003) que la cultura investigativa es "toda manifestación cultural, organizaciones, actitudes, valores, objetos, métodos y técnicas, todo en relación con la investigación, así como la transmisión de la investigación o pedagogía de la misma". La educación superior forma para ejercer una profesión; una buena orientación al escogerla, permite aprendizajes pertinentes (Lara, 2011). La formación contable debe ser innovada, cambiar lo convencional y adentrarse en una nueva educación que incite más a situaciones de análisis crítico para sentar posiciones frente a diversas problemáticas (Ordoñez, 2008). La contabilidad ha estado expuesta a la cooperación de muchos pensadores de gran valor, ellos realizan significativo aporte a través de diversas escuelas que definen el objeto de estudio y generan bases 
teóricas para mejorar la estructura del conocimiento contable (Coelho da Rocha, 2004). Estos profesionales, requieren habilidades: intelectuales, investigativas, personales, de comunicación y creatividad, para que sean los profesionales que requiere la sociedad (Cano Morales, Arango Henao, \& Zapata Morales, 2004).

\section{METODOLOGÍA, MATERIAL Y MÉTODOS}

La investigación se sustenta en el paradigma cualitativo-cuantitativo de tipo descriptivo de carácter etnográfico, debido a que buscó caracterizar a estudiantes y docentes en cuanto al concepto que tienen respecto a la investigación y la manera como promueven la formación investigativa a partir de los procesos de enseñanza, aprendizaje, competencias y hábitos para el desarrollo de actividades orientadas a la investigación. La etnografía- parafraseando a (Guber, 2.000), como método y como enfoque para comprender desde el punto de vista de los actores las percepciones acerca de las acciones que se realizan en la academia que se orientan a la apropiación de conocimiento y a generar relaciones que conducen a una práctica investigativa. De este modo, hacer etnografía consisten en "establecer relaciones, seleccionar a informantes, transcribir textos, establecer genealogías, trazar mapas de área llevar un diario... es como tratar de leer (en el sentido de "interpretar un texto")". Lo particular de este tipo de investigación es la simultaneidad de los procesos que vuelven realidad de los elementos teóricos descritos, encontrando los datos que constituyen la evidencia del tipo de relaciones planteadas (Geertz, 1994).

Este tipo de investigación centra la atención en la descripción, interpretación y comprensión de los aspectos culturales relacionados con la formación para la investigación y la forma de apropiación de conocimiento por parte de los actores involucrados; como lo expresa (Martínez, 2.000) "intenta hacer una aproximación global de las situaciones sociales para explorarlas, descubrirlas y comprenderlas de manera inductiva", al conocer e identificar las prácticas pedagógicas, actividades de aula y comportamientos que favorecen el desarrollo del pensamiento, en el contexto académico desde una visión integral, dando las propias significaciones e interpretaciones de acuerdo con las información recogida en el trabajo de campo. Dice (Guber, 2.000) al respecto: "una buena interpretación es aquella que no los malinterpreta".
En lo cuantitativo, parafraseando a (Orozco, 1997,) citado por (Monje Alvarez, 2011, pág. 12) esta se sustenta en que para el positivismo lo que interesa es la cuantificación, medición; es con esta que se logra medir y cuantificar ciertas repeticiones para llegar a establecer tendencias, formular nuevas hipótesis y construir teorías, fundadas en el conocimiento cuantitativo; como no es posible contar todo se inventó la estadísticas para acercarse a la totalidad pero por medio de muestras. Este estudio toma como unidad de análisis a las Universidades Santo Tomás, Autónoma de Bucaramanga, Cooperativa de Colombia, Corporación Universitaria de Ciencia y Desarrollo (UNICIENCIA) y Unidades Tecnológicas de Santander.

Participan cuatrocientos sesenta y un (461) estudiantes, treinta y dos (32) profesores y cinco (5) decanos. Se hizo un "mapeo", cómo se dice en la investigación cualitativa, para poder lograr un acercamiento al objeto de estudio y así, indagar el problema planteado. De esta manera se identificó el actor cooperante, en palabras de Schwartz y Jacobs (1984), citado por (Sandoval Casilimas, 1996), denomina "cartografía Social". La muestra fue tomada por juicio y conveniencia de las investigadoras porque hubo limitación al elegir los participantes. De manera progresiva se fueron tomando los datos, hasta cuando hubiera saturación de la información y por tanto estuvo sujeto a la dinámica de la recolección de información derivada de los mismos hallazgos.

A continuación se presentan las técnicas e instrumentos de recolección de información:

a) Entrevistas semi-estructuradas, dirigidas a decanos y directores de programas, se buscó hacer que hablaran de lo que saben, piensan y creen sobre la calidad académica.

b) Revisión documental, se recopila normatividad sobre educación superior en Colombia y sustento teórico para las variables estudiadas.

c) Cuestionarios en la modalidad de encuesta, se diseñan dos cuestionarios y se aplican a profesores y estudiantes.

d) Los datos suministrados por los participantes se clasificaron por variables, indicadores, categorías y se ubicaron en una matriz para facilitar el análisis.

El análisis de la información se realiza teniendo en cuenta las percepciones más relevantes de cada uno de los grupos participantes; se compararon e interpretaron a la luz de los ele- 
mentos teóricos del interaccionismo simbólico y la investigación en el contexto académico. Se trianguló la información con los contrastes desde la información de las entrevistas, la observación y los datos obtenidos, se verificó la información con los fundamentos teóricos que orientaron el trabajo. La investigación optó por un enfoque de tipo comprensivo como lo expone (Sandoval, 1996).

La captación a través de la interpretación y el diálogo, del sentido de lo que el otro o los otros quieren decir con otras palabras o silencios, con sus acciones o sus inmovilidades, sino también la posibilidad de construir generalizaciones que permitan entender los aspectos comunes a muchas personas y grupos humanos en su proceso de producción y apropiación de la realidad social y cultural en que desarrollan su existencia.

\section{RESULTADOS}

La contabilidad ha sido considerada como una disciplina empírica, antes la formación contable era entendida de carácter técnico; ahora la tendencia del entorno económico cambia y se le añade el componente científico, esto hace que la contabilidad se ubique en la sociedad del conocimiento. Los estándares internacionales de educación contable, definen las mejores prácticas para la educación en este campo de conocimiento (Suáres Henao \& Contreras Patiño, 2012, págs. 175-186). Surge una transformación de la universidad, como lo plantea Sandoval (1988) citado por (López Jímenez, 2001) "la universidad se entiende como una institución que crean conocimiento, que adapta a las condiciones nacionales o regionales los paradigmas teóricos y tecnológicos generados por los circuitos de la ciencia internacional, propone nuevos paradigmas, nuevas tecnologías y al tiempo, lleva a cabo la formación de los sucesores de las comunidades científicas presentes en ellas, por medio de la constitución de una comunidad académica o docente".

Las instituciones objeto de estudio presentan diferencias en la filosofía como: principios cristianos, economía solidaria, propiedad del Estado y la trilogía estética compuesta por la visión del mundo, del ser humano y la educación; tienen particularidades que las identifican; sin embargo convergen en impartir educación con pertinencia social; los procesos académicos se orienten a formar para la investigación y hacer investigación en sentido estricto. Ver figura 1.

Figura 1. Integración de la investigación en los currículos.

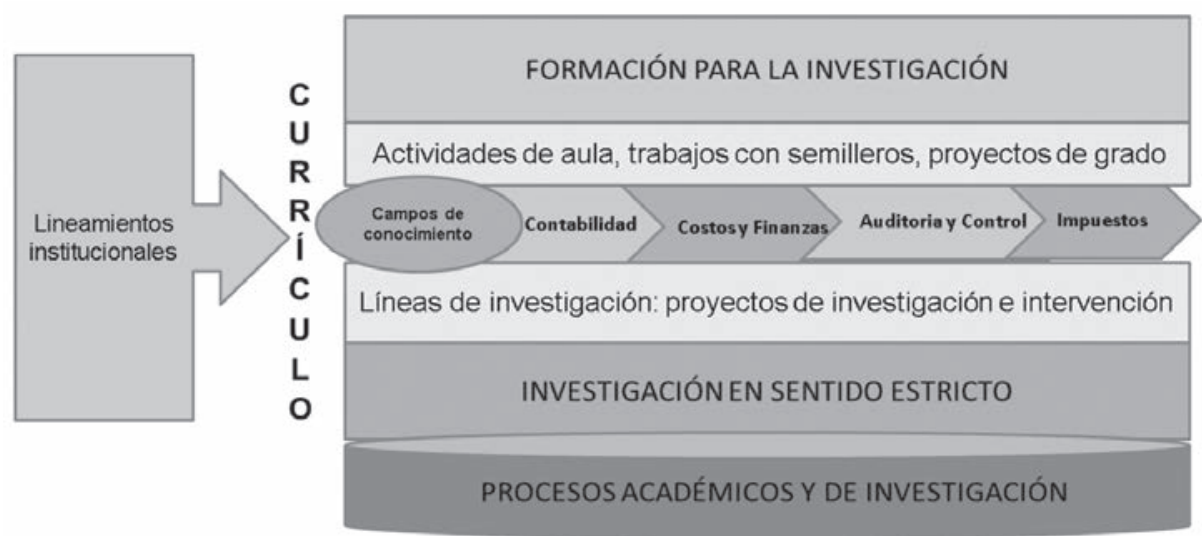

Fuente. Elaboración Propia.

Mencionan que la investigación es una construcción colectiva que sigue lineamientos y políticas institucionales donde trazan directrices para definir líneas, grupos de investigación, áreas y campos de conocimiento con temáticas de interés y problemas concretos. Los actores son semilleros y grupos de investigación, jóvenes investigadores, auxiliares de investigación y estudiantes con proyectos de grado. Consideran que a investigar se aprende investigando, requieren estrategias de enseñanza-aprendizaje que motive la investigación y desarrollen com- 
petencias para la lectura, escritura, consultas de fuentes bibliográficas, técnicas para la producción de texto, lectura comprensiva y crítica, habilidades discursivas y disposición para participar en eventos académicos e investigativos. Ver figura 2.

Figura 2. Estrategias que fomentan la investigación en el aula.

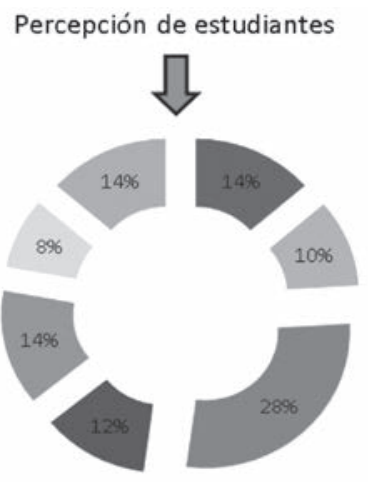

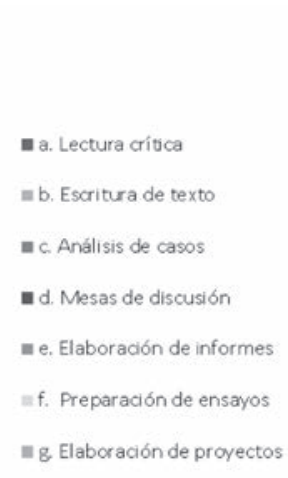

Percepción de Profesores

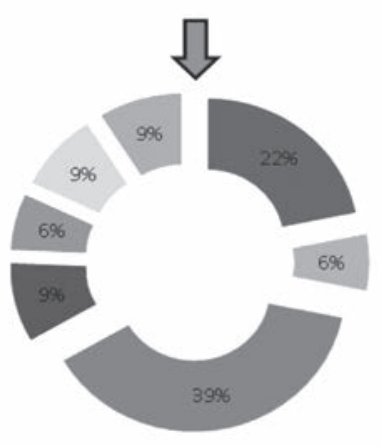

Fuente. Elaboración Propia.

Los estudiantes expresaron que la mejor forma de llegar a la práctica investigativa es mediante la interacción estudiante-profesor, esta se contrasta con lo manifestado por los docentes, dicen que la investigación es una acción propia de la práctica pedagógica, las mismas estrategias que facilitan el aprendizaje fomenta la investigación, estas se definen en el plan de estudio. La percepción de los profesores y estudiantes, seleccionan el análisis de casos como factor dominante de las estrategias, a través de estos se llega a desarrollos prácticos con aplicación teórica, de modo que facilita la interpretación y comprensión de fenómenos que intervienen en las alternativas de solución, con el fin de llegar a nuevo conocimiento; la lectura crítica está en segundo lugar, manifiestan que es una forma de expresar lo que comprenden e interpretan desde una postura argumentativa para elaborar otros concep- tos orientados a teorizar

Desde la mirada cuantitativa, el 53\% de los estudiantes expresan que la investigación los conduce a la generación de conocimiento, a obtener información, ganar desarrollo y crecimiento; es consistente con lo que menciona el $50 \%$ de los profesores sobre las mismas variables. Los estudiantes reconocen la importancia de la investigación, sin embargo, el $86 \%$ no participan en estas actividades, los profesores la reconocen y se vinculan a uno varios espacios de investigación, solo un $4 \%$ no participa. Las capacidades y habilidades que contribuyen en procesos de investigación, las valoran en una escala de uno a cinco, en donde el valor de uno es el más bajo y cinco el más alto, califica según la percepción que tiene cada uno las potencialidades. Ver Cuadro I. 
Cuadro I. Valoración de capacidades y habilidades.

\begin{tabular}{|c|c|c|c|c|c|c|c|c|c|c|c|c|}
\hline \multirow{3}{*}{ Capacidades y habilidades } & \multicolumn{6}{|c|}{ Estudiantes } & \multicolumn{6}{|c|}{ Docentes } \\
\hline & \multicolumn{6}{|c|}{ Escala de valoración } & \multicolumn{6}{|c|}{ Escala de valoración } \\
\hline & 1 & 2 & 3 & 4 & 5 & Total & 1 & 2 & 3 & 4 & 5 & Total \\
\hline Lectura comprensiva & 10 & 30 & 141 & 221 & 59 & 461 & 0 & 0 & 4 & 14 & 14 & 32 \\
\hline Redacción de texto & 16 & 53 & 136 & 196 & 60 & 461 & 1 & 1 & 9 & 9 & 12 & 32 \\
\hline Uso de reglas ortográficas & 21 & 40 & 125 & 171 & 104 & 461 & 3 & 0 & 8 & 7 & 14 & 32 \\
\hline Manejo de herramientas y medios & 9 & 19 & 110 & 215 & 108 & 461 & 0 & 5 & 4 & 10 & 13 & 32 \\
\hline Facilidad de expresión & 31 & 54 & 140 & 159 & 77 & 461 & 1 & 1 & 1 & 13 & 16 & 32 \\
\hline Manejo de auditorio o público & 76 & 95 & 130 & 105 & 55 & 461 & 0 & 0 & 1 & 12 & 19 & 32 \\
\hline
\end{tabular}

Fuente. Elaboración Propia.

Los estudiantes expresan que la formación académica contribuye al desarrollo de competencias encaminadas a generar cultura investigativa, los factores expuestos anteriormente son parte de ello. La mayoría de estudiantes concentran la valoración en cuatro. Los profesores califican los mismos factores, sobresale la valoración cuatro y cinco, porque consideran que como docentes alcanzan mayor potencialidades.

La lectura y escritura la perciben como componente de aprendizaje e investigativo; el 6\% revelan que tiene hábito de lectura, se recrean con esta actividad y sienten motivación. El 81\% de los profesores creen que la lectura y escritura contribuye en el proceso académico, es un factor que motiva, permite recrearse con los des- cubrimientos y profundización que realizan en diversos temas. Tanto docentes como estudiantes manifiestan que los temas de preferencia y sobre lo que más leen son cultura, economía, finanzas, impuestos y auditoría; un poco menos deportes. Añaden que en la labor académica realizan trabajos de clase y de corte investigativo; los profesores comentan que deben preparar las clases, cumplir con proyectos de investigación y trabajos. Exponen que consultan diversas fuentes, lo más importante es encontrar los temas requeridos, aunque se preocupan que las fuentes abordadas sean confiables y respondan a las exigencias de los protocolos y tipos de trabajos que elaboran. Ver figura 3.

Figura 3. Fuentes consultadas en el desarrollo de trabajos.

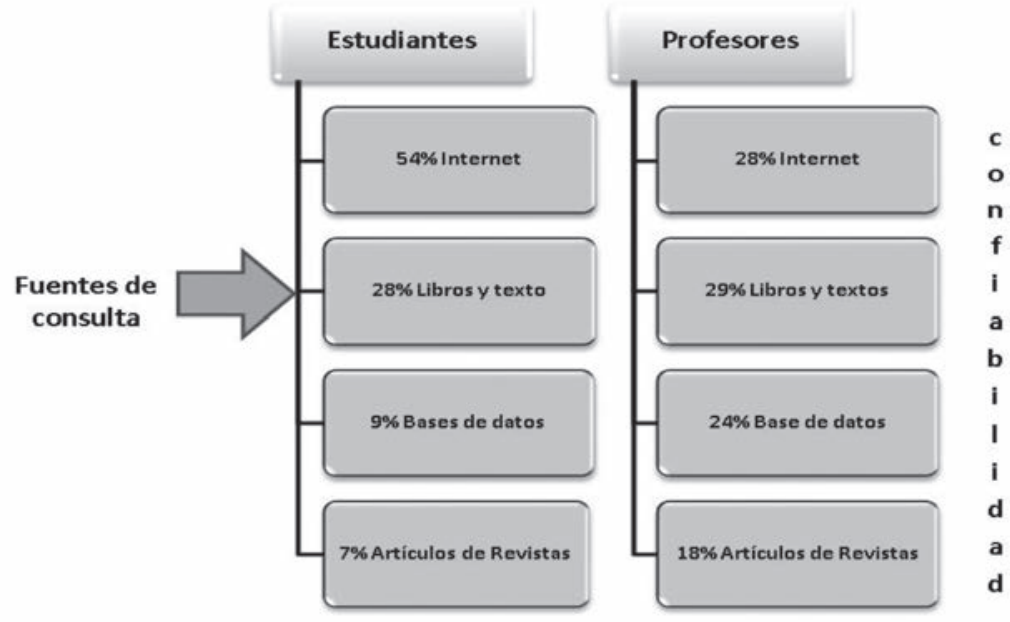

Fuente. Elaboración Propia. 
Los participantes expresan que en realidad el desarrollo de trabajos es por compromiso, consultan y leen lo necesario para la elaboración del trabajo, los hábitos de lectura son insuficientes. El 25\% de los estudiantes comentan que no han leído ningún libro en los últimos años y el $52 \%$ han leído solo entre uno y dos, el 55\% dedican de una a dos horas a leer y escribir para el desarrollo de trabajos asignados. El $44 \%$ de los profesores leen de uno a dos libros, el 25\% leen entre cinco y seis libros; el 34\% dedican de una a dos horas a leer y escribir y el $41 \%$ disponen de cinco a seis horas para el desarrollo de esta actividad. Expresan que las instituciones están construyendo cultura, que falta camino por recorrer, notan que existe compromiso y están abiertos para motivar la formación con pertinencia y calidad en el campo contable, el fin es alcanzar la responsabilidad social de la educación por ser un servicio público.

\section{CONCLUSIONES}

La investigación contable se genera en los ambientes académicos mediante actividades de clase, la función docente permite que el estudiante llegue a inquietarse por la indagación para incursionar en espacios investigativos y posteriormente establecer una relación entre docencia y práctica profesional; esto denota la importancia de formar para la investigación y hacer investigación en sentido estricto; de modo que se aporte a la gestión de conocimiento contable. La educación contable debe ocuparse de impartir las posibilidades para desarrollar diez aprendizajes o saberes fundamentales: aprender a conocer, aprender a hacer, aprender a vivir juntos e impartir el conocimiento, aprender a leer, aprender a hablar, aprender a pensar, aprender a escuchar, aprender a observar, aprender a escribir; aprender a ser (Cano Morales, Arango Henao, \& Zapata Morales, 2004). Los procesos académicos debe sentar las bases para la investigación con el fin de renovar y construir nuevos conocimientos; a investigar se aprende investigando.

Se destaca la importancia de conformar líneas de investigación, grupos de investigación, áreas y campos del conocimiento contable como base para proponer proyectos de investigación e interacción, en aras de la innovación y desarrollo tecnológico en las organizaciones. Plantean estrategias para promover la lectura, escritura para el desarrollo de procesos investigativos en la academia; desde esta perspectiva el estudiante genera hábitos de indagación que lo conducen al mundo de la investigación con una postura crítica, argumentativa y discursiva. Por ser la contabilidad una ciencia aplicada, en las actividades de aula se enfatiza en el análisis de casos debido al carácter técnico. La contabilidad es una disciplina con limites difusos, aparece tensionada en torno a dos núcleos conflictivos: el primero es el estatuto científico como una disciplina empírico social y en segundo orden las prioridades de las presiones entórnales que exigen que se cumplan objetivos mediatos e inmediatos para enfrentar requerimientos de práctica aplicada, es decir la tecnología dominada por usos sociales (León Martínez, 2008).

La dinámica de los negocios, exige que el profesional contable sea protagonista de cambio e incursione en comunidades científicas, se disponga a hacer desarrollos en la ciencia contable para trascender las fronteras del conocimiento. El contador público es un trabajador del conocimiento, entonces los procesos de formación deben ser de la misma connotación. Esto no sucede. A cambio de formar trabajadores del conocimiento se forman trabajadores de servicios. Los contadores públicos no "vendemosvenden" servicios, es cierto que vendemos un intangible similar, pero es conocimiento. Simplificamos la complejidad contable, así nosotros mismos veamos esa habilidad como sencilla y la subvaloremos (Sánchez, 2004). La cultura investigativa en esta comunidad académica es baja, falta compromiso con la profesión, las universidades saben que la educación se sustenta en tres pilares: docencia, investigación y extensión o proyección social y asumen el deber de propiciar una construcción colectiva.

En la formación de un contador público juega un papel importante la integralidad del individuo, un aspecto fundamental es el desarrollo de competencias como lo expresa (Sánchez, 2004) caracterizadas por:

a) Enfocar el desempeño profesional y no los contenidos de los cursos.

b) Mejorar la relevancia de lo que se aprende.

c) Facilitar la integración de contenidos aplicables al trabajo.

d) Generar aprendizajes aplicables a situaciones complejas.

e) Favorecer la autonomía de los individuos.

f) Transformar el papel de los docentes hacia una concepción de facilitar y provocar.

Los roles expuestos anteriormente pueden aportar elementos de discusión importante en torno a temas como las políticas de investiga- 
ción de las universidades, los grupos de investigación contable las políticas editoriales de las revistas y la visión de la institucionalidad contable y el mundo profesional (Rueda Delgado, 2014). La responsabilidad social de las instituciones de educación superior, modelos pedagógicos, enseñanza de la contabilidad fundada desde lo conceptual, práctico y ético, y pertinencia de la educación en épocas de cambio, orientada hacia la construcción de una cultura investigativa en la formación contable son líneas aptas para seguir investigando. No hay que perder de vista, que es en la academia donde se fundamenta el perfil del contador público, este se define a partir de las necesidades del contexto y los referentes de la educación, se buscan los saberes esenciales que los caracteriza; se propende por el desarrollo de las dimensiones del ser que se refiere al saber conocer, saber hacer, saber ser y saber vivir juntos en la diversidad.

\section{REFERENCIAS}

Cano Morales, A. M., Arango Henao, J. B., \& Zapata Morales, M. A. (2004). ¿Está la contaduría pública inmersa en la sociedad del conocimiento? Contaduría, 112, 13.

Coelho da Rocha, L. F. (2004). Aportes sobre la doctrina científica del neopatrimonialismo contable. Contabilidad \& Auditporía, 129, 144.

Geertz, C. (1994). Descripción densa: hacia una teoría interpretativa de la cultura. En la interpretación de las culturas. Barcelona: Gedisa.

Guber, R. (2.000). La etnografía. Método, campo y Reflexibilidad. Pag. 13. Bogotá: Norma.

Lara, M. E. (2011). Fundamentos de investigación. Un enfoque por competencias. Bogotá: Afahomega.

León Martínez, G. (2008). La resignificación de los contextos del conocimiento a proposito del plan de formación contable. Perspectiva critica de la Contabilidad. Reflexiones y criticas contables alternas al pensamiento único, 127-158.

López Jímenez, N. (2001). La de-construcción curricular. Bogotá: Cooperativa Editorial Magíterio.

Martínez, M. (2.000). La investigación cualitativa Etnografica en educación.
Monje Alvarez, C. A. (2011). Metodología de la investigación cuantitativa y cualitativa. Guía didactica. Neiva: Universidad Surcolombiana.

Ordoñez, L. (2008). Contara el adiestramiento contable: invitación a la rutra epistemológica en la formación del Contador Público. Perspectivas críticas de la.

Restrepo, B. (2003). Conceptos y aplicaciones de la investigación formativa y criterios para evaluar la investigación científica en sentido estricto.

Ritzer, G. (2002). Teoría sociológica moderna. Madrid: Mc Graw Hill.

Rueda Delgado , G. (2014). El papel de las publicaciones en medio de las tensiones entre la investigación académica y la práctica contable. Cuadernos de contabilidad número 38, 357-361.

Sánchez, W. (2004). Educación contable, calidad educativa, Ecaes y pedagogía. Contabilidad \& Auditoria, 141, 155.

Sandoval Casilimas, C. (1996). Investigación Cualitativa. Bogotá: ICFES.

Sandoval, C. (1996). Investigación Cualitativa . Bogotá: ICFES.

Suáres Henao, A. V., \& Contreras Patiño, I. V. (2012). La formación integral del contador público colombiano desde la expectaiva internacional: un analisis a partir de los estandares internacionales de educación IES. Gestión \& Desarrollo. 9(1), 175-186.

Unigarro, M. (1999). Introducción a las teorías educativas contemporáneas.

Wright, S. (2005). Antrhopology of organizations. Routledge, Londres. 